Abb. 1 Um Erkrankungen durch den dosha vata (leicht, kalt und trocken) zu beruhigen werden die Prinzipien von schwer, heiß und ölig eingesetzt. Quelle: NiDerLander/Adobe Stock

\title{
Einführung in den Ayurveda
}

Der Ayurveda ist ein großes traditionelles Medizinsystem aus Indien - Heutzutage ist der Ayurveda hochaktuell, weil sich unter seiner Logik alle Erkrankungen einordnen und behandeln lassen

Hedwig Gupta

Der Ayurveda ist das mit Abstand größte und bekannteste System der traditionell indischen Medizin (TIM). Seine Wurzeln liegen in den altindischen Vedas, die nach langer mündlicher Tradition vor mehreren Tausend Jahren niedergeschrieben wurden. Bis heute bezieht man sich im Ayurveda auf eine klassische Literatur, die vor ca. 2000 Jahren geschrieben wurde. Die wichtigsten und ältesten Lehrbücher sind die unter dem Begriff der „Großen Drei“ zusammengefassten Carakasamhita, Sushrutasamhita und Ashtangahridaya.

Heute ist der Ayurveda ein von der WHO anerkanntes Medizinsystem, das in vielen Ländern Asiens gleichberechtigt neben der
Schulmedizin praktiziert wird. Dort wird er im Rahmen eines 5-jährigen Universitätsstudiums mit dem Abschluss „BAMS“ gelehrt. Nach weiteren 3 Jahren kann man sich an indischen Universitäten zum Facharzt im Ayurveda spezialisieren (MD ay. bzw. MS ay.). Dies schließt mit einer größeren wissenschaftlichen Arbeit ab. Nach weiteren 5 Jahren kann ein ayurvedischer Facharzt auch einen PhD im Ayurveda erlangen.

\section{Grundlagen des Ayurveda \\ Fünf Elemente - pancamahabhutas}

Die Basis des ayurvedischen Verständnisses der materiellen Welt sind die fünf Elemente (pancamahabhutas). Sie sind die Wirkprin- zipien, die sich in allem wiederfinden lassen: den Geweben, der Ernährung, den Heilkräutern etc. Die gesamte Evolution entsteht durch immer neue, veränderte Kombinationen der fünf Elemente. Im Einzelnen sind sie

- Erde (prithivi) - Prinzip der Masse, Substanz und Stabilität.

- Wasser (ap/jala) - Prinzip der Kohäsion und Flüssigkeit

- Feuer (tejas) - Prinzip der umwandelnden Energie

- Luft/Wind (vayu) - Prinzip der Beweglichkeit

- Himmel/Äther (akasha) -Prinzip des freien Raumes 


\section{Zusammenfassung}

Der Ayurveda ist ein WHO-anerkanntes, großes traditionelles Medizinsystem aus Indien. Er fußt auf einer differenzierten Anatomie und Physiologie und kann alle Störungen und Erkrankungen ganzheitlich in einem logischen System von Ätiologie und Pathologie erfassen und diagnostisch einordnen. Der Ayurveda hat einen breit gefächerten, differenziert einsetzbaren Therapiebaum.

Heutzutage ist der Ayurveda hochaktuell, weil sich unter seiner Logik alle Erkrankungen einordnen und behandeln lassen und sich eine ayurvedische Therapie ohne Schwierigkeiten mit anderen Medizinsystemen kombinieren lässt.

\section{Drei Bioenergien - tridoshas}

Das Funktionieren des Individuums auf körperlicher, sinnlicher, geistiger und emotionaler Ebene wird von den tridoshas reguliert. Die doshas sind aus den fünf Elementen zusammengesetzt, und zwar wie folgt:

- vata - aus Himmel und Luft, d.h. den Prinzipien von Raum und Bewegung

- pitta -aus Feuer und ein wenig Wasser, d. h. vor allem dem Prinzip der umwandelnden Energie

- kapha -aus Wasser und Erde, d.h. aus den Prinzipien von Kohäsion und Masse Schon aus dem Wissen der Zusammensetzung der tridoshas aus den fünf Elementen können die Aufgaben der tridoshas erfasst werden.

- vata ist verantwortlich für Bewegung, Kontrolle, Impulse, Taktung. Es wird beschrieben als trocken, kalt, leicht, subtil, beweglich, klar und rau.

- pitta ist zuständig für Umwandlung, Verdauung, Stoffwechsel und Färbung. Seine Eigenschaften werden beschrieben als leicht fettig, heiß, durchdringend, flüssig, sauer, beweglich und scharf.

- kapha sorgt für Körpermasse, Stabilität und Kühlung. kapha ist schwer, kalt, weich, fettig, süß, unbeweglich und schleimig.

An den Eigenschaften der doshas kann man sie im Individuum erkennen und grob quantifizieren. Die Eigenschaften vermitteln aber auch die Wirkungen der Substanzen: so wird Kälte zur Zunahme der kalten doshas, vata und kapha, führen, Wärme zur Abnahme der kalten doshas.
Die doshas befinden sich in einem dynamischen Gleichgewicht. Sie kontrollieren sich gegenseitig. Wenn ein dosha aus der Kontrolle entweicht, werden automatisch alle Vorgänge im Körper verändert.

Die Harmonie der drei doshas ist Ursache für Gesundheit. In diesem Zustand erhalten sie den Körper. Ihre Dysbalance hingegen Ursache von Erkrankung. In diesem Zustand können sie den Körper zerstören.

\section{vata}

ist der wichtigste der drei doshas. Körperlich sorgt vata für Taktung, Impuls und Zirkulation und unterhält damit die tragenden Bestandteile des Körpers, geistig-emotional führt er zur Ausbildung von Begeisterung, Intuition und Schnelligkeit. vata entsteht im Dickdarm.

Typische Symptomatik von vata-Übereizung sind Schmerz, Verstopfung, Blähung und unregelmäßiger Stuhl. Auch ein plötzliches, schubweises Auftreten von Beschwerden, Unruhe und Schlaflosigkeit werden durch vata hervorgerufen.

\section{pitta}

verursacht alle Umwandlungsprozesse, Färbung und Wärmebildung. Es ist für die Ausbildung von Intelligenz und Klarheit zuständig. pitta wird im Oberbauch gebildet. Typische Symptome einer pitta-Störung sind Brennen, Fieber, Rötung, Entzündung, Blutung oder Ulzerierung. Wut und Ärger zeigen eine Reizung von pitta auf emotionaler Ebene.

\section{kapha}

zeigt sich in Zusammenhang mit Stabilität, Kraft und Kühlung. kapha bildet Aspekte wie das Langzeitgedächtnis, Verständnis und Mut. Der Hauptsitz von kapha ist im Brustraum.

Wird kapha gereizt, entstehen Symptome wie Jucken, Schwellung, Verdickung, Verschwartung, Verschleimung, Ödeme, Schwere oder Nässe.

\section{Körperliche Konstitution}

Das funktionelle Gleichgewicht der doshas, das Gesundheit bedeutet, ist im Ayurveda kein Absolutum, sondern von Person zu Person verschieden. Anders als in der Schulmedizin, in der für alle Menschen gültige Normbereiche entwickelt sind, betont Ayurveda das individuell richtige Gleichgewicht. Wo das Gleichgewicht der verschiedenen doshas liegt, welcher dosha also bei der jeweiligen Person im gesunden Zustand stärker hervortritt, das bestimmt die schon intrauterin bestehende Konstitution des Menschen.

\section{Sieben Grundgewebearten - saptadhatu}

Der Ayurveda ist zwar ein energetisches Medizinsystem, aber er beschreibt dennoch auch die Bestandteile des Körpers. Die Gewebe (dhatus) sind zunächst unbewegte Struktur. Nur über die Wirkung der doshas werden sie zum Leben und zur Funktion erweckt.

Sieben Grundgewebearten sind im Ayurveda beschrieben:

- „Plasma“ ( rasa): klare Flüssigkeiten im Körper,

- „Blut“ (rakta): rote Flüssigkeiten im Körper,

- „Muskel“ ( mamsa): fleischartige Organe,

- „Fett“ (medas): weiches Bindegewebe,

- „Knochen“ (asthi): hartes Bindegewebe,

- „Mark“ (majja): Knochenmark oder Nervengewebe und

- „Samen“ (shukra): progenetisches Gewebe.

Gesundheit bedeutet, dass auch alle Gewebe in ihrer Menge, Eigenschaften und Funktionen normal sind. Krankheit ist Abnormalität in einem der Aspekte.

\section{Immunität - ojas}

Das ojas ist so etwas wie das Immunsystem auf körperlicher und psychomentaler Ebene. Von der Menge und dem Funktionieren des ojas hängen Leben und Gesundheit ab.

ojas ist eine feine Substanz und wird als Essenz aller Gewebe gebildet.

Da man im Ayurveda davon ausgeht, dass eine Krankheit nur dann entstehen kann, wenn der Mensch nicht stark genug ist, die doshas im Gleichgewicht zu halten, ist das ojas, das dafür eine Art Puffersystem dar-

\section{Die Harmonie der drei doshas ist Ursache für Gesundheit. Ihre Dysbalance ist die Ursache} von Erkrankung. 
stellen, wichtig in Therapie und Prophylaxe von Erkrankungen: Solange genug ojas vorhanden ist, erlaube es den doshas nicht, die Gewebe zu erkranken.

In der Therapie von allen chronischen Krankheiten, bei Schwäche und wiederkehrenden Erkrankungen sowie nach Unfall wird man im Ayurveda grundsätzlich das ojas stärken, sodass eine Erkrankung in $\mathrm{Zu}$ kunft abgewehrt werden kann.

\section{Biokanalsystem - srotamsi}

Der Körper besteht nicht nur aus einem Haufen Zellen und Organen. Es heißt in den klassischen Schriften, dass der gesamte Körper von Systemen von Kanälen durchzogen wird. Über diese Kanäle ist Informationsund Stoffaustausch möglich. Durch die Kanäle ziehen doshas, Plasma und Blut und sorgen für Ernährung und Funktion des Körpers. Die Kanäle heißen srotas (Singular) bzw. srotamsi (Plural).

Sie sind im gesamten Körper vorhanden: in jedem Gewebe und in jeder einzelnen Zelle. Die srotamsi sind nicht nur passive Öffnungen, sondern erlauben den selektiven Transport von Stoffen.

Man unterscheidet 13 srotas-Systeme: Es gibt drei srotas-Systeme für die vitalen Aufgaben wie Herzschlag und Atmung, Wasserhaushalt und Nahrungsaufnahme, sieben srotamsi für sieben Grundgewebearten und drei für die drei Haupt-Ausscheidungsprodukte. Gewebe und srotamsi hängen funktionell eng zusammen.

\section{Ausscheidungsprodukte - malas}

Jedes Gewebe produziert Abfallprodukte im Verlauf des Stoffwechsels. Auch diese müssen in harmonischer Weise produziert und ausgeschieden werden. Jedes Zu-viel, Zuwenig, Zu-schnell, Zu-langsam, Zu-dick, Zudünn oder Zu-geruchsintensiv ist ein Zeichen für eine Stoffwechselstörung.

Die drei wichtigsten Ausscheidungsprodukte sind Stuhl, Urin und Schweiß.

\section{Stoffwechsel - agni}

Nahrungsaufnahme und Stoffwechsel sind das zentrale Thema der ayurvedischen Physiologie. Wenn der Stoffwechsel nicht in seinen regelmäßigen Bahnen verläuft, führt das unabdingbar zu Erkrankung, weil das doshas, Gewebe und Biokanäle (srotamsi) aus ihrem Gleichgewicht bringt.

Eine ayurvedische Diagnose ohne Beurteilung des Stoffwechsels (agni) ist unvoll- ständig. Eine ayurvedische Therapie sollte immer den Stoffwechsel regulieren. Ohne diese Maßnahme sind alle anderen therapeutischen Bemühungen entweder nutzlos oder von kurzem Erfolg.

\section{Stoffwechselschlacken - ama}

Folge von einem unsauberen Brennen des Stoffwechsels (agni) ist, dass die Nahrung nicht vollständig verdaut wird. Wie bei einem Motor, der nicht richtig eingestellt ist, entstehen Schlackenstoffe, un- bzw. fehlverdaute Metabolite, das sogenannte ama.

ama zirkuliert durch den Körper. Wegen seiner Klebrigkeit bleibt es an den Stellen hängen, an denen die innere Wand der Biokanäle (srotamsi) verändert ist. Dort setzt sich ama ab und sorgt für Blockaden der Kanäle.

Da die doshas auch durch die Kanäle ziehen, verbinden auch sie sich mit ama.

Die doshas und die Nähressenz können wegen ama die Gewebe nicht mehr richtig ernähren. Die Fehlernährung führt zur Fehlfunktion der Gewebe, so dass auch diese in den Krankheitsprozess einbezogen werden.

\section{Diagnostik im Ayurveda}

Im Ayurveda gibt es immer eine Doppeldiagnose

1. die Diagnose des Patienten (rogi-pariksha)

2. die Diagnose der Krankheit (roga-pariksha)

Daraus wird eine differenzierte Therapie abgeleitet und eine Prognose hinsichtlich der Therapiedauer und -schwere erstellt.
- Konstitution des Kranken - geistig und körperlich - Immunkraft (ojas)

- Stoffwechsel (agni)

- Konstitution der Krankheit

- Name (wenn vorhanden)

- dosha

- Gewebe und deren Abfallprodukte

- Ort der Erkrankung: Organ und

Biokanal (srotas)

Abhängig von dieser differenzierten Diagnose wird die Prognose gestellt sowie die individuell korrekte Therapie ausgewählt, die typischerweise aus verschiedenen Säulen des ayurvedischen Therapiekonzeptes kombiniert besteht.

\section{Ayurvedische Therapie}

Die Allgemeine Therapie hat zwei Hauptziele:

1. Gesundheitsförderung: Dies ist das wichtigste Ziel, zu dem auch alle Therapie von Erkrankungen am Ende wieder hinführen sollte. Dazu werden Ernährungs- und Verhaltens-empfehlungen individuell adaptiert, Stimmungsstabilisatoren und Stärkungsmittel verschrieben und Methoden zur Rejuvenation eingesetzt.

2. Heilung von Erkrankungen: Entsteht trotz der Gesundheitsförderung eine Erkrankung, wird die Therapie zur Heilung von Erkrankungen notwendig. Diese teilt sich in verschiedene Richtungen:

- Die „Göttliche Therapie“ gibt es für kausal nicht verständliche Erkrankungen. Diese werden als vom Schicksal verur-

\section{„Derjenige, der eine Krankheit nicht mit einem Namen benennen kann, sollte sich nicht schämen, weil nicht alle Krankheiten mit einem Namen ausgezeichnet sind.“}

Ca. Sa. Su. 18: 44

Der Grund dafür ist, dass der gleiche veränderte dosha verschiedene Krankheiten hervorrufen kann.

Wichtig für die ayurvedische Diagnose ist das Festhalten der folgenden Aspekte:

- Umgebung und Jahreszeit

- Land, Jahreszeit, Lebensabschnitt sacht angesehen. Als kausale Therapie hierfür werden individuell passende Rituale wie Mantren, Gebete, Edelsteine, Wallfahrten etc. eingesetzt. Diese Therapieformen werden nicht universitär gelehrt, sondern typischerweise von Geistlichen oder Astrologen empfohlen. 


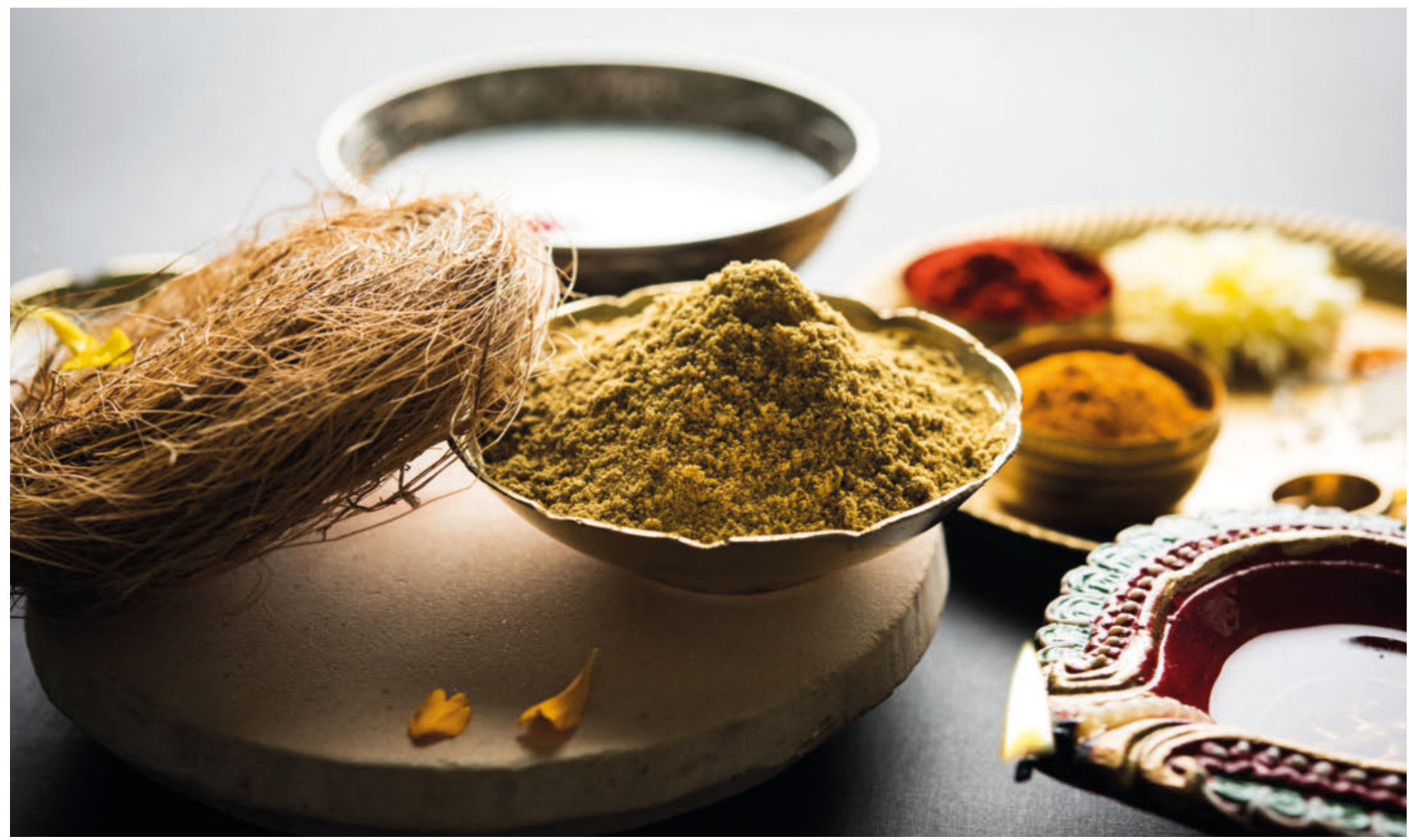

Abb. 2 Die ayurvedische Phytotherapie ist reichhaltig. Hunderte von klassisch beschriebenen Pflanzen kommen zum Einsatz. Quelle: StocklmageFactory/Adobe Stock

- Die „Rationale Therapie“ ist die zentrale Therapiesäule für kausal interpretierbare Erkrankungen. Sie hat drei Hauptzweige mit verschiedenen Ansätzen: - doshas ausleiten (samshodhana), die innere Reinigung mit Ausleiten der veränderten doshas mit den klassischen pancakarma-Methoden.

- doshas beruhigen (samshamana), die veränderten doshas werden über innerliche wie äußerliche Behandlungsansätze wieder in ihr Gleichgewicht gebracht. Diese beiden Behandlungszweige werden weiter unten im Text genauer beschrieben.

- Chirurgische Interventionen: Zu klassischen Zeiten war der Ayurveda auch auf chirurgischem Fachgebiet führend. Bis heute spielen parachirurgische Therapien wie Schröpfen oder Behandlungen von Fisteln oder Hämorrhoiden noch eine bedeutende Rolle.

- Die „Psychotherapie“. Die ayurvedische Psychotherapie umfasst Aspekte wie Coaching, Gedankenhygiene und Verhaltenstherapie. Sie wird stets in Kom- bination mit den anderen Säulen der Therapie eingesetzt.

\section{dosha-ausleitende Therapie - samshodhana}

samshodhana bedeutet reinigende Therapie. Ziel ist das Ausleiten der veränderten doshas. Dahinter steht die Rationale, dass man die doshas, wenn sie nur mäßig erregt sind, mit beruhigenden Methoden wieder ausgleichen kann. Wenn sie aber stärker verändert sind, können sie so nicht mehr in den ausgeglichenen Zustand kommen. Diese veränderten doshas müssen ausgeleitet werden. Im Anschluss daran bilden sich im Körper neue doshas, die ein neues, gesundes Gleichgewicht bilden.

Daher heißt es von den reinigenden Techniken, dass sie die Wurzel der Krankheit ausräumen, so dass die Krankheit nicht wieder auftritt, während bei beruhigender Behandlung die doshas leichter erneut ins Ungleichgewicht kommen können.

Das gilt natürlich nur dann, wenn im Anschluss an die reinigende Behandlung, die die Krankheit auslösenden Ernährungs- und Ver- haltensweisen verändert werden, so dass die Ursache der dosha-Erregung verschwindet.

Die reinigende Therapie ist unter dem Namen „pancakarma“ bekannt. „pancakarma“ setzt sich aus den Sanskritbegriffen „panca“ (fünf) und „karma“ (Handlung) zusammen. Es handelt sich um fünf reinigende Techniken, nämlich:

- Therapeutisches Erbrechen (vamana),

- Abführen (virecana),

- Einlauf (basti),

- Nasenbehandung (shirovirecana) und

- Schröpfen (raktamokshana).

Diesen therapeutischen Methoden geht immer eine vorbereitende Behandlung voraus und eine Nachbehandlung folgt.

Die Vorbehandlung soll den Körper weich machen und die gereizten doshas von der Stelle der Erkrankung lösen und in die jeweiligen Hauptsitze der doshas (Magen, Dünndarm und Dickdarm) bewegen. Von dort werden die doshas über die jeweils indizierte Reinigungstechniken ausgeleitet.

Im Anschluss werden die Nachbehandlungen angewandt, um die harmonische Bildung der neuen doshas zu fördern. 


\section{dosha-beruhigende Therapie - samshamana}

Die dosha-beruhigenden Therapien gleichen die doshas über den Einsatz gegensätzlicher Eigenschaften aus. Da kapha z.B. schwer, kalt und ölig ist, wird es über Behandlungen beruhigt, die leicht, heiß und trockene Eigenschaften haben. Am häufigsten sind Erkrankungen durch den dosha vata. Dieser ist leicht, kalt und trocken. Um ihn zu beruhigen werden die Prinzipien von schwer, heiß und ölig eingesetzt. Die kann äußerlich wie innerlich erfolgen.

\section{Äußerliche Anwendungen}

Diese beruhen auf dem Prinzip von Ölbehandlungen und Wärmeanwendungen.

Beide sind Teil des klassischen pancakar$m a$ als vorbereitende Therapie, aber auch als beruhigende Therapie selbständig einsetzbar.

\section{Ölbehandlung (snehana)}

Es gibt viele verschiedene Formen von Massagen, Einstreichungen, Ölgüssen, die in Abhängigkeit der Art und Lokalisation der Erkrankung verschrieben werden.

\section{Wärmeanwendung (svedana)}

Klassischerweise werden

- 10 aktive, ohne äußerliche Hitzezuführung funktionierende Wärmetherapien (z.B. warme Kleidung, körperliche Übungen etc.) und

- 13 Formen der Wärmeanwendung mit einer äußeren Hitzequelle (z.B. Dampf, warmes Wasser, trockene Wärme etc.) beschrieben.

Sie können nur einem Teil des Körpers oder dem ganzen Patienten zugeführt werden. Typischerweise werden die äußerlichen dosha-beruhigenden Techniken für eine Zeit von mehreren Tagen bis Wochen täglich eingesetzt, um eine tiefe Wirkung zu entfalten.

\section{Innerliche Anwendungen}

Hier werden innerliche Medikamente verschrieben. Meist kommen Phytotherapeutika zur Anwendung, aber auch tierische Produkte wie Milch, Honig, Muscheln, Perlen etc. und mineralische Produkte wie Erdpech, Eisen, Gold, Edelsteine etc.

Sie werden z. B. in Form von Saft, Abkochungen, Pulver, Vergärungen, Tees oder Kaltauszügen gegeben. Die Logik folgt ei- nem ayurvedischen Einordnungssystem, das die Chemie übe Geschmack, Eigenschaften, Geschmack nach der Verdauung, Wirkpotenz und spezifische Wirkrichtung beschreibt. Zunächst wird stets der Stoffwechsel (agni) eingestellt. Das Ziel ist entweder Organ- oder dosha-bezogen.

Die Phytotherapie ist reichhaltig (Abb. 2). Hunderte von klassisch beschriebenen Pflanzen kommen zum Einsatz. Viele davon sind auch aus moderner Sicht in Hinblick auf ihre antioxidative, analgetisch, antiinflammatorische, krebsreduzierende u. a. Potenz untersucht. Die indische Regierung hat eine Materia medica mit aktuellen Forschungsergebnissen herausgegeben.

Die ayurvedische Therapie ist typischerweise multimodal. Ein Therapiekonzept beginnt stets mit der Therapie der Ursachen, die meist in der Ernährungs- und Lebensweise der Erkrankten gesehen wird. Dazu werden die doshas ausgeglichen, der Stoffwechsel gestärkt, die Stoffwechselschlacken aus dem System entfernt und ggf. die doshas gereinigt.

\section{Günstige Indikationen für eine ayurvedische Behandlung}

Der Ayurveda ist ein umfassendes Medizinsystem und kann daher alle Erkrankungen diagnostizieren und behandeln.

Heute empfiehlt sich der Einsatz des Ayurveda insbesondere bei Erkrankungen, die multifaktoriell verursacht sind, wie chronische oder psychosomatische Erkrankungen, unspezifische und funktionelle Störungen, Schmerzerkrankungen, immunologische und rheumatische Geschehen, Erschöpfungs- und Reizzustände, unerfüllter Kinderwunsch oder degenerative Erkrankungen.

Man kann die ayurvedische Therapie auch sehr gut mit Ansätzen anderer Medizinsysteme kombinieren.

Interessenkonflikt: Die Autorin erklärt, dass keine Interessenkonflikte bestehen.

Online zu finden unter

http://dx.doi.org/10.1055/a-1056-8573
Weiterführende und Hintergrundliteratur

1 Sharma PV. Caraka Samhita. Varanasi: Chaukambha Vasvabharati; 1994

2 Sharma PV. Sushruta Samhita. Varanasi: Chaukambha Vasvabharati; 1999

3 Murthy SKR Vagbhata's Ashtanga Hridayam. Varanasi: Chowkhambha Krishnadas Academy; 2009

4 Gupta HH. Einführung in den Ayurveda. 2. Aufl. Asperg: Verlag für Vedische Wissenschaften; 2017

5 Gupta HH. Ayurveda-vijnana I-IV. Asperg Verlag für Vedische Wissenschaften: 2013-2019

6 Mittwede M. Der Ayurveda. Stuttgart: Haug; 1998

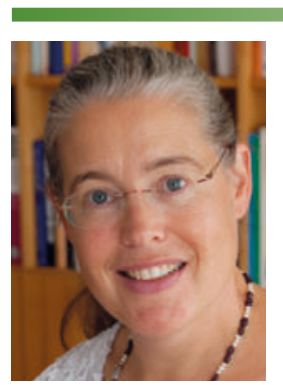

Dr. med. Hedwig H. Gupta

Fachärztin für Orthopädie und Rheumatologie, Ayurveda, therapeutischer Yoga, Akupunktur, manuelle Medizin

Lammstr. 1

71679 Asperg

www.vidya-sagar.de

info@dr-gupta.de

Hedwig H. Gupta, Fachärztin für Orthopädie und Rheumatologie. Nach dem Studium der Humanmedizin Postgraduiertenstudium von Ayurveda, Yoga und Sanskrit an der BHU, Indien. Autorin von Fachbüchern und Fachartikeln. Vorstandsvorsitzende der DÄGAM (Deutsche Ärztegesellschaft für Ayurveda-Medizin) und Vorständin der DeGYT (Deutsche Gesellschaft für Yogatherapie). Leiterin der vidya-sagar-Akademie für Ayurveda und Yogatherapie. 